\title{
A High Cyano Content Indacene as a Stable Organic Electron Acceptor
}

\section{Key words}

electron acceptor

indacenes

nitriles
Significance: The authors used rational design to target the reported structure, compound $\mathbf{1}$. The indacene framework was stabilized by the high degree of cyano functionalization without compromising ease of reduction, which has been an issue with indacenes stabilized by benzannulation. The high cyano content also lowered the LUMO to $-5.8 \mathrm{eV}$. Cyclic voltammetry shows four reversible single electron transfers, and UV/Vis shows a band gap of $\sim 1.9 \mathrm{eV}$.
Comment: The synthesis began from a previously reported compound (C. Adams et al. Inorg. Chim. Acta 2011, 366, 44), produced $330 \mathrm{mg}$ of pure $\mathbf{3 a}$, and should be scalable. Compound $\mathbf{3 a}$ was found to be generally stable both as a solid and in solution, possibly due to the low-lying LUMO. 